\title{
PROPOSED ACTIVE-STANDBY SWITCH OF THE DIGITAL OUTPUT FOR THE REDUNDANT ATO SYSTEM
}

\author{
Zhang Jianming $^{1, a}$, Luan Qingwen ${ }^{2, b}$ and Yan Zengwei ${ }^{3, c}$ \\ ${ }^{1}$ Beijing Traffic Control Technology Co., Ltd; 100070; Beijing \\ ${ }^{2}$ Beijing Traffic Control Technology Co., Ltd; 100070; Beijing \\ ${ }^{3}$ Beijing Traffic Control Technology Co., Ltd; 100070; Beijing \\ ajianming.zhang@bj-tct.com, ${ }^{\mathrm{b}}$ qingwen.luan@bj-tct.com, ${ }^{\mathrm{c}}$ zengwei.yan@bj-tct.com
}

Keywords: Redundant ATO, Digital Output and Active-Standby Switch

\begin{abstract}
By taking into account the hot standby structure of the redundant ATO system, the paper discusses the key technologies (including self-inspection feedback and fault clearing) for the digital output in the system and proposes a new method of the digital output in the redundant ATO system to discover any fault of its output channel in a timely manner and carry out the active-standby switch to make the system reliable.
\end{abstract}

\section{Introduction}

With development of the major cities, the rail transit becomes the most important urban public transport. The ATO system (automatic operation control system) provides the automatic control of the train through the improved algorithm to ensure less interferences of the driver with the train, so it plays a vitally important role in smaller operation interval and greater transport capacity. As such, the ATO system is catching ever-increased attention of the people for the reliability. The mainstream ATO systems are believed to apply the hot standby structure to improve the reliability at present.

The hot standby-based ATO system has two similar systems of which one operates and the other is in a hot standby status more often than not. The working primary system will automatically switch to the standby one when any fault is detected. The active-standby switch involves that of the dual host server and the dual system communication and output. The paper discusses switch of the dual system output including self-inspection and switch of the fault exported from the channel.

\section{Introduction to the System}

The ATO system is comprised of digital input, host server and digital output in general. However, for the purpose of the hot standby-based ATO system, redundancy is required for these units. By taking into account the restrictions (including space and wiring) of the vehicle, the digital input and output both serve as an individual node at a side of the vehicle, so the same channel shall be applied for the redundant digital input and output before the vehicle is provided. The digital output allows for simultaneous acquisition of the signals in the dual system, while the digital output signal shall be exported from the primary system with the standby one in a waiting state. The primary system which detects any output fault has the active-standby switch to ensure service of the standby system as the primary one before the digital output.

The digital output shall meet the following two conditions for the active-standby switch:

(1) The system detects the status of its output channel in a timely and proper way to provide the basis for the active-standby switch;

(2) When the system fails, it is possible to clean any fault of the primary system effectively to prevent any negative impact upon the normal output of the primary system. 


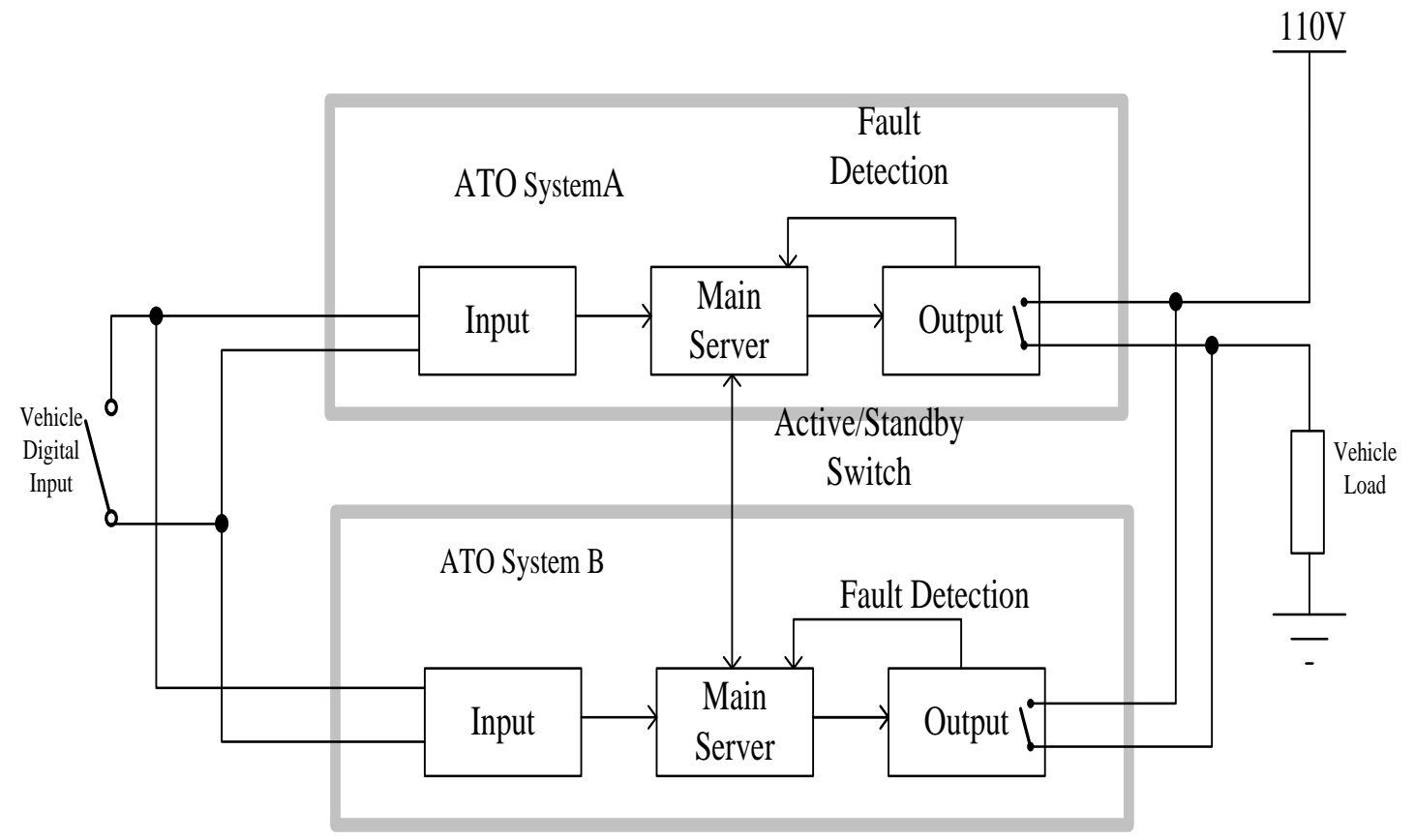

Figure 1 Structure of the Redundant ATO System

\section{Key Technologies}

\subsection{Fault Detection}

The fault detection represents the basis of the active-standby switch. In this sense, no active-standby switch is allowed before effective detection of any fault in the system.

In general, the ATO system provides for the vehicle the relay-based digital output signals through the dry contacts. The indirect feedback is very common at present. That is, the relay is applied for the output through a contact and feedback through another contact (see Fig. 2). In most cases, there is no problem with the feedback acquisition, but either poor closing of the contact or fault of the relay's connecting rod may cause inconsistent action of both contacts with the consequent failed or erroneous detection of the system's fault.

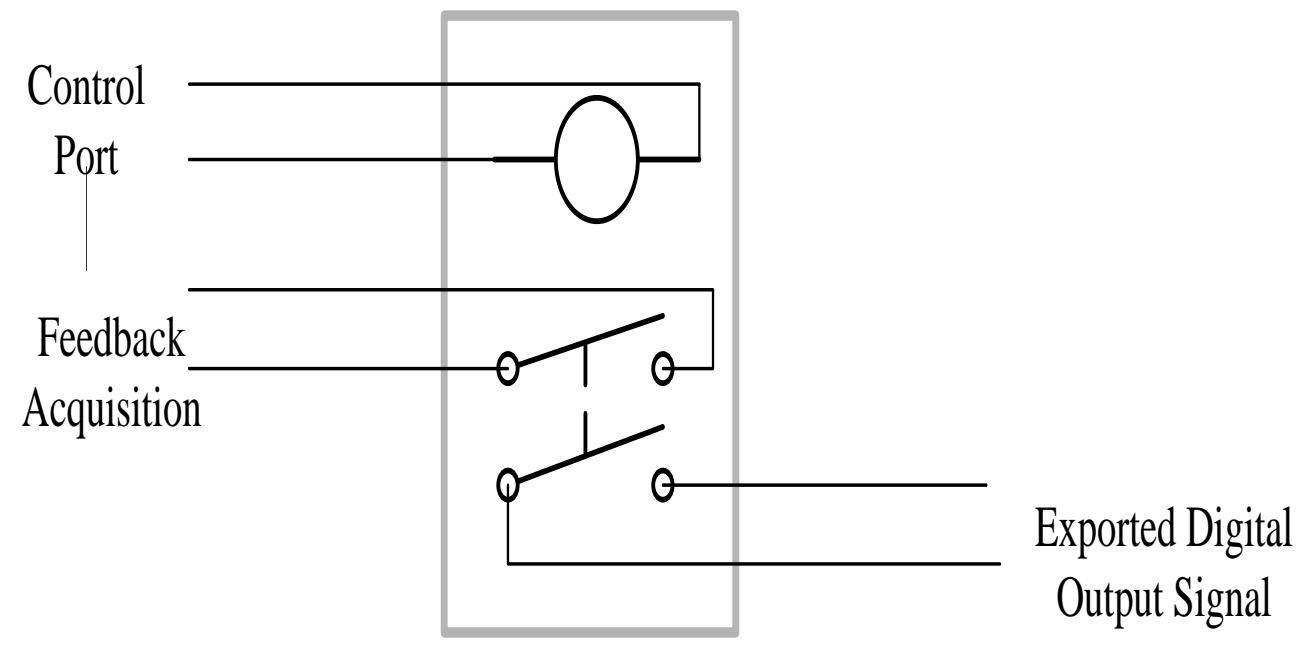

Figure 2 Way of the Indirect Output Feedback

Application of the direct feedback (at the side of the digital output provided by the redundant ATO system for the vehicle) may reflect the output status directly. For instance, the Hall sensor is applied in a series form for the acquisition (see the following figure): 


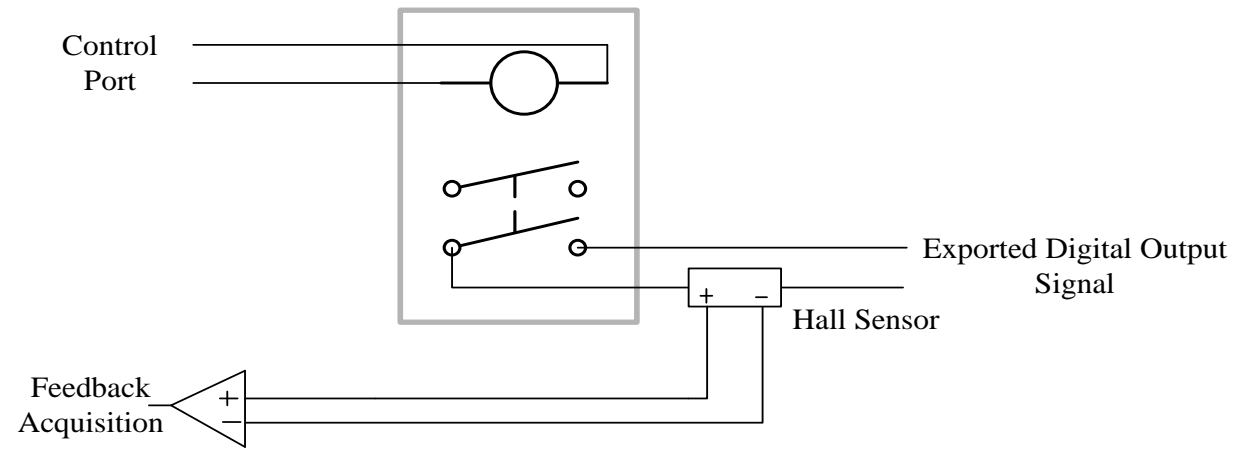

Figure 3 Application of the Hall Sensor for the Acquisition

The Hall sensor is developed in the form of the magnetic field on the basis of the Hall effect. An ordinary Hall sensor has four lead terminals of which two are connected in a series form to the output circuit of the redundant ATO system to input the bias current for the Hall element and the other two are intended to output the Hall voltage. It is possible to produce the Hall current when a circuit is formed between both output terminals. The exported current may be connected to the input stage of the operational amplifier to check if the external switch is closed by means of the level of the voltage exported from the amplifier. The redundant ATO system provides for the vehicle the digital signals through the dry contacts, so the current flow ranges from several milliamperes to several amperes with the different connected load of the vehicle. In this case, to ensure such an extensive range and the accuracy, the Hall sensor shall have a greater dimension which is not suitable for the project-specific application. In this paper, the fault is detected by means of feedback acquisition of the contacts through the isolated power source.

In most cases, the $110 \mathrm{~V}$ or $24 \mathrm{~V}$ power $\mathrm{DC}$ is applied for acquisition of the digital output from the redundant ATO at a side of the vehicle. Feedback acquisition of the AC signals is possible by applying the characteristics of the capacitor featuring no DC but AC. The AC signals may be produced through the 555 or low-frequency crystal oscillator, and the roughly $1 \mathrm{~K}$ frequency is usually applied to avoid the excessive interference signals. The AC signals are processed for no DC through the capacitor before being connected in parallel to the digital output on the redundant ATO. The feedback signals remain in an AC form and are detected through the diode and acquired in a feedback form through the driving optoisolator.

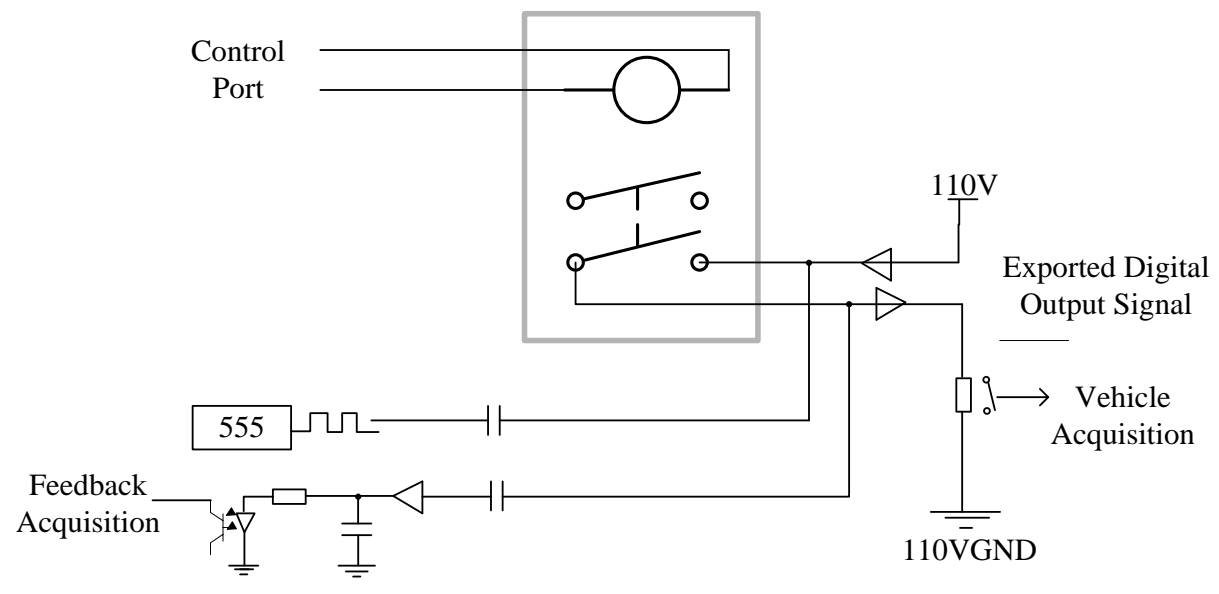

Figure 4 Way of the Direct AC Acquisition

In general, acquisition is made at a side of the vehicle by connecting $110 \mathrm{~V}$ to the anode of the redundant ATO's digital output whose cathode is connected to the anode of the relay coil with its cathode connected to the GND of the $110 \mathrm{~V}$ power. The AC signals have the following circuit: The AC signals are coupled through the capacitor into the $110 \mathrm{~V}$ power and return to a terminal for feedback acquired signals through the $110 \mathrm{~V}$ power's internal resistance, relay coil and lower-end capacitor with the consequent acquisition of the feedback signals, so a diode is required for isolation 
of the redundant ATO's digital output before the vehicle is provided. That is because a detector diode is provided in the feedback acquisition circuit and a diode at a side of the digital output for the vehicle. Both diodes are placed in a reverse form in the AC circuit to prevent coupling of the AC signals into the feedback acquisition circuit.

The capacitor has the blocking characteristics to ensure no impact of the $110 \mathrm{~V}$ power DC upon the feedback acquisition circuit with possible superposition of the power at both ends, so the capacitor shall have a withstand voltage of greater than $110 \mathrm{~V}$ and a safety capacitor is required for the derating purpose.

\subsection{Fault Clearing}

Any defected fault shall be cleared to prevent any negative impact upon the normal output of the primary system. The digital output faults are categorized into the following: (1) Poor closing of the output which causes failed acquisition of the signals from the main server at a side of the vehicle; (2) Output adhesion which causes the main server to export any unexpected signal.

The redundant ATO-based dual system outputs are connected in parallel. In this sense, any fault featuring poor closing of the digital output contacts may not affect the other system output only when one system is disconnected with the power. For the purpose of both system, the main server functions in active-standby switch through the relay preferred circuit. Upon the fault, the primary system will automatically give up control of the preferring relay. The power for driving the digital output also has control of the final output signals through any contact on the preferring relay. The exported power will be automatically cut off when the system give up the preferring relay.

Considering parallel connection of both systems, any fault featuring the digital output adhesion will cause a failure to clear the final output signals even if the system is disconnected with the power. For this very reason being, the digital output of the single system shall apply series connection of two contacts on the relay and be controlled by two terminals. The final digital output signals are closed only when the control signals are exported from both terminals to ensure clearing of the two contacts which are not adhesive simultaneously. The AC signals are detected at both sides of the intermediate contact which is provided for both of the series connected contacts to check if contact adhesion of the single relay occurs through the feedback acquisition 1 and feedback acquisition 2.

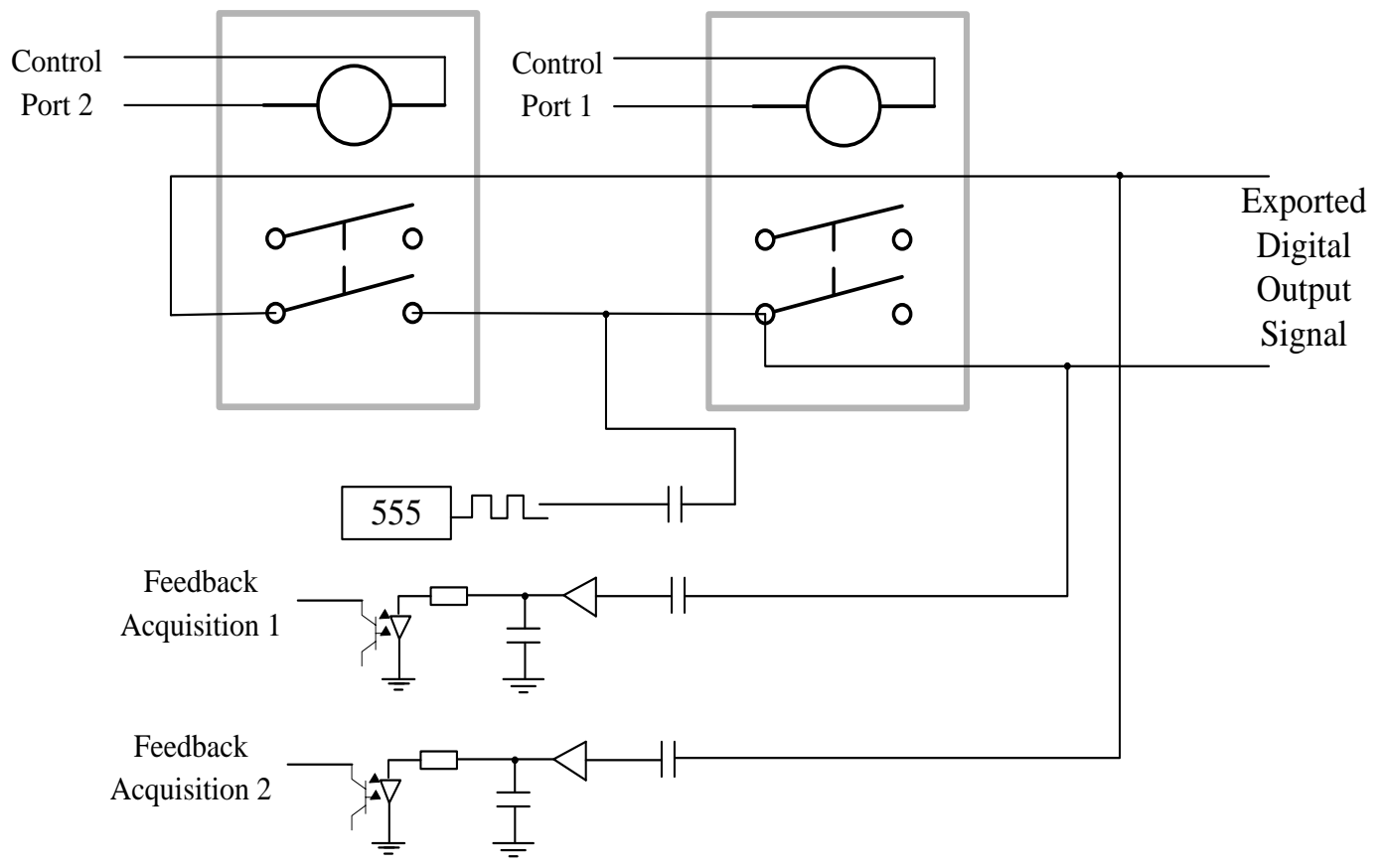

Figure 5 Way of the Contact Series Connected Feedback Acquisition 


\subsection{Verification Test}

For the purpose of validation of the test equipment, first of all, verification of the proper output logic is required: Upon the simultaneous energization of both systems, the system A will obtain the priority to become the primary system. The manual active-standby switch is required and both systems shall have the output through the relay. As shown in the parameters of the selected relay, the contacts will last for 100 milliseconds or so in response to energy storage of the relay coil when it is disconnected with the power. The switch takes about 20 milliseconds between the systems and the switch time causes no chattering of the contacts to ensure the seamless switch.

Simulation of the fault featuring poor closing of the single system's digital output contacts: First of all, manual disconnection with the output is required for a system. In the event that no feedback signal is acquired in a period (200ms) after the system output, then it shall belong to the fault of the channel output and the system is considered to give up the priority. By detecting any fault of the primary system, the standby system serves as the primary one to continue exporting the signal in the circuit. A delay of the period is possible for exporting of the signals in the circuit and it is tolerable for the redundant ATO system.

Simulation of the fault featuring short-circuit of the single system's digital output: First of all, manual short-circuit treatment of the output is required for a system. First of all, self-inspection is required when both system are energized. Considering series output of the single system, application of one exported way after another is required for the self-inspection. First, with the excited coil at the control terminal 1, feedback acquisition 1 may have the feedback signals, but feedback acquisition 2 doesn't ; then, with excitation loss of the coil at the control terminal 1 and the excited coil at the control terminal 2, feedback acquisition 2 may have the feedback signals, but feedback acquisition 1 doesn't. If any contact adhesion of the relay (e.g. relay 2) occurs, feedback acquisition 2 may also have the feedback signals when the feedback coil 1 is excited. In this case, the system is considered to have the failed self-inspection and give up the active-standby priority.

\section{Conclusion}

The digital output is one of the most important step for the ATO system. The ways as shown in the paper support the effective detection of any fault of the system's digital output and full clearing of the failed primary system through the series and parallel connected structures to ensure no affected operation of the system.

The design is intended to verify feasibility of the feedback and improvement the feedback is base on the test result in the next step to develop an engineering system.

\section{References}

[1] Meng Jun, Li Kejian and Li Liang: Design and Possibility of the ATP Determined ATO Redundancy Structure, Railway Signalling \& Communication, Vol. 51(4) (2015), P. 13-16

[2] Li Liang, Yin Xunzheng and Meng Jun: Design and Possibility of the Safety Platform Determined ATO System Redundancy, Railway Computer Application, Vol. 23(2) (2014), P. 32-35

[3] Gao Jixiang and Zheng Junjie: Study of the Reliability and Safety Indicators of the Hot Standby Computer Interlocking System, Journal of Beijing Jiaotong University, Vol. 22 (5) (1998), P. 73-77

[4] Jiang Jianhua: Technical Study and Specific Possibility of the Hot Standby System, Microcomputer Applications, Vol. 20(3) (2004), P. 7-9

[5] Wang Jiangjiang, Li Zhiqiang and Zhao Liang: Study of the Hot Standby System concerning the Active-Standby Switch, Railway Signalling \& Communication, Vol. 51 (2) (2015)

【Note】Scientific and Technical Committee of Beijing plans to provide the financial support of the "Development and Demonstrative Application of the Smallest System Equipment for the FAO-based Rail Transit (D141100000814001)". 